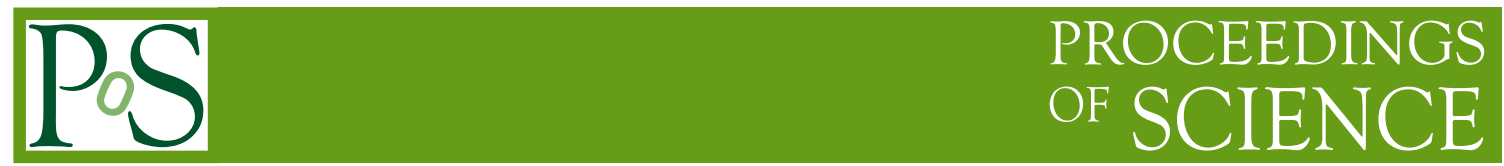

\title{
Charm hadron physics at BESIII
}

\author{
Xiaokang Zhou*† \\ University of Science and Technology of China, Hefei, China \\ E-mail: rhouxkdustc.edu.cn
}

The BESIII Experiment at the Beijing Electron Positron Collider (BEPCII) has accumulated the world's largest $e^{+} e^{-}$collision samples at $\psi(3770)$ peak, at $4.178 \mathrm{GeV}$ for $D_{S}^{*+} D_{S}^{-}$decay and at the $\Lambda_{c}^{+} \bar{\Lambda}_{c}^{-}$pair mass threshold, which allow us to study decays of charmed mesons and baryons in a uniquely clean background. In this report, recent results in charm hadronic decay at BESIII will be presented.

The 15th International Conference on Flavor Physics \& CP Violation 5-9 June 2017

Prague, Czech Republic

* Speaker.

${ }^{\dagger}$ on behalf of the BESIII collaboration. 


\section{Introduction}

The analyses presented in this report are based on two data samples collected by the BESIII detector[四], installed at the double-ring BEPCII collider (IHEP, Beijng, P.R.C). One is s the largest $e^{+} e^{-}$annihilation sample in the world to date, $2.92 \mathrm{fb}^{-1}$, at the $3.773 \mathrm{GeV}$ center-of-mass energy, where $e^{+} e^{-} \rightarrow \psi(3770) \rightarrow D \bar{D}$ is mainly produced. Another one is the unique and largest, $0.5 \mathrm{fb}^{-1}$, at the center-of-mass energy of $4.599 \mathrm{GeV}$ which is the $\Lambda_{c}^{+} \bar{\Lambda}_{c}^{-}$mass threshold.

We make use of double-tag technique initially used by MARK III [వ] to identify the $D \bar{D} / \Lambda_{c}^{+} \bar{\Lambda}_{c}^{-}$. In this technique, the yields of single tags (ST), where one $D / \Lambda_{c}$ is reconstructed in the tag modes, and double tags (DT), where both $D / \Lambda_{c}$ mesons are reconstructed, are determined. In this report, $\bar{D}^{0}$ is reconstructed in one of three tag modes: $\bar{D}^{0} \rightarrow K^{+} \pi^{-}, K^{+} \pi^{-} \pi^{0}$ and $K^{+} \pi^{-} \pi^{-} \pi^{+}$, while $D^{-}$is reconstructed in one of six tag modes: $D^{-} \rightarrow K^{+} \pi^{-} \pi^{-}, K^{+} \pi^{-} \pi^{-} \pi^{0}, K_{S}^{0} \pi^{-}, K_{S}^{0} \pi^{-} \pi^{0}, K_{S}^{0} \pi^{-} \pi^{-} \pi^{+}$ and $K^{+} K^{-} \pi^{-}$, while $\Lambda_{c}^{+}$is reconstructed in one of twelve tag modes: $\Lambda_{c}^{+} \rightarrow p K_{S}^{0}, p K^{-} \pi^{+}, p K_{S}^{0} \pi^{0}$, $p K_{S}^{0} \pi^{+} \pi^{-}, p K^{-} \pi^{+} \pi^{0}, \Lambda \pi^{+}, \Lambda \pi^{+} \pi^{0}, \Lambda \pi^{+} \pi^{-} \pi^{+}, \Sigma^{0} \pi^{+}, \Sigma^{+} \pi^{0}, \Sigma^{+} \pi^{+} \pi^{-}$and $\Sigma^{+} \omega$. The hadronic decays are identified using the beam-constrained mass $M_{B C}=\sqrt{E_{\text {beam }}^{2}-p^{2} c^{2}}$, where $E_{\text {beam }}$ is the beam energy and $p$ is measured momentum of $D^{-} / \bar{D}^{0} / \Lambda_{c}^{+}$and energy difference $\Delta E=E_{\text {beam }}-E$, where $E$ is the measured energy of $D^{-} / \bar{D}^{0} / \Lambda_{c}^{+}$. Throughout this report, the inclusion of charge conjugated processes is implied, unless otherwise explicitly mentioned.

In this proceeding, four measurements of $D$ hadronic decays and four measurements of $\Lambda_{c}$ hadronic decays are reported.

\section{2. $D$ hadronic decays}

\subsection{Absolute branching fraction (BF) of $D^{0} \rightarrow K_{S}^{0} / K_{L}^{0} \pi^{0}\left(\pi^{0}\right)$ and $y_{C P}$ measurement (preliminary)}

Non-leptonic $\mathrm{D}$ decays and their strong phases have been of great interest as they are essentially related to the studies of CP violation (CPV), $D \bar{D}$ mixing and SU(3) symmetry breaking effects in charm physics. As first pointed out by I.I.Bigi and H.Yamamoto [B]], the decay rates of $D \rightarrow K_{S}^{0} \pi$ and $D \rightarrow K_{L}^{0} \pi$ are not the same because of the interference of the Cabibbo-favored (CF) component $D \rightarrow K^{0} \pi$ with the doubly Cabibbo-suppressed (DCS) component $D \rightarrow \bar{K}^{0} \pi$. Since the interference of $K^{0}$ with $\bar{K}^{0}$ is opposite for $K_{S}^{0}$ and $K_{L}^{0}, \mathscr{B}_{D \rightarrow K_{S}^{0} \pi^{\prime} s}$ and $\mathscr{B}_{D^{0} \rightarrow K_{L}^{0} \pi^{\prime} s}$ should not in general be equal. The scale of the asymmetry is set by the DCS factor $\tan ^{2} \theta_{C} \approx 0.05$, where $\theta_{C}$ is the Cabibbo angle.

In BF measurement of $D^{0} \rightarrow K_{S}^{0} / K_{L}^{0} \pi^{0}\left(\pi^{0}\right)$, the quantum correlation should be considered [四]. In the absence of $\mathrm{CPV}$ and $D \bar{D}$ mixing, the $\mathrm{CP}$ eigenstate $\mathrm{BF}$ is extracted by $\mathscr{B}_{C P \pm}=\frac{1}{1 \mp C_{f}} \frac{N_{C F, C P \pm} / \varepsilon}{N_{C F}}$, $\left(C_{f} \equiv \frac{2 \operatorname{rcos} \delta}{1+r^{2}}\right)$, where $N_{C F, C P \pm}\left(N_{C F}\right)$ denote the DT (ST) yields, $\varepsilon$ is the signal efficiency, $C_{f}$ is a correction factor derived from $r$ and $\delta, r$ is the ratio of the DCS to CF amplitudes for $D^{0}\left(\bar{D}^{0}\right)$ decays to the same $\mathrm{CF}$ final state, and $\delta$ is the strong phase difference between the two amplitudes. $C_{f}$ can be measured using the $\mathrm{CP} \pm \mathrm{ST}$ events and $\mathrm{CF}$ vs. $\mathrm{CP} \pm \mathrm{DT}$ events. The asymmetries of the BFs of $D^{0} \rightarrow K_{S}^{0} / K_{L}^{0} \pi^{0}\left(\pi^{0}\right)$ can be determined by $R\left(D^{0} \rightarrow K_{S}^{0} / K_{L}^{0} \pi^{0}\left(\pi^{0}\right)\right)=\frac{\left.\mathscr{B}_{K_{S}^{0}}^{0} \pi^{0} \pi^{0}\right)^{-\mathscr{B}} K_{L}^{0} \pi^{0}\left(\pi^{0}\right)}{\mathscr{B}_{K_{S}^{0}} \pi^{0}\left(\pi^{0}\right)^{+\mathscr{B}} K_{L}^{0} \pi^{0}\left(\pi^{0}\right)}$. The results can be found in Table $\mathbb{L}$, which agree well with the measurement of CLEO-c[[]]. 
Table 1: BFs and asymmetries of $D^{0} \rightarrow K_{S}^{0} / K_{L}^{0} \pi^{0}\left(\pi^{0}\right)$. The uncertainties in each tag are statistical only. The first uncertainties in average results are statistical and the second are systematic.

\begin{tabular}{lccc}
\hline \hline tag modes & $\mathscr{B}_{K_{S}^{0} \pi^{0}}(\%)$ & $\mathscr{B}_{K_{L}^{0} \pi^{0}}(\%)$ & $R\left(D^{0} \rightarrow K_{S}^{0} / K_{L}^{0} \pi^{0}\right)$ \\
\hline$K \pi$ & $1.218 \pm 0.042$ & $1.062 \pm 0.038$ & $0.069 \pm 0.025$ \\
$K \pi \pi \pi$ & $1.225 \pm 0.037$ & $0.987 \pm 0.036$ & $0.107 \pm 0.023$ \\
$K \pi \pi^{0}$ & $1.254 \pm 0.029$ & $0.959 \pm 0.028$ & $0.133 \pm 0.018$ \\
Average & $1.237 \pm 0.020 \pm 0.033$ & $0.993 \pm 0.019 \pm 0.029$ & $0.109 \pm 0.012 \pm 0.018$ \\
\hline \hline tag modes & $\mathscr{B}_{K_{S}^{0} \pi^{0} \pi^{0}}(\%)$ & $\mathscr{B}_{K_{L}^{0} \pi^{0} \pi^{0}}(\%)$ & $R\left(D^{0} \rightarrow K_{S}^{0} / K_{L}^{0} \pi^{0} \pi^{0}\right)$ \\
\hline$K \pi$ & $1.025 \pm 0.049$ & $1.299 \pm 0.081$ & $-0.118 \pm 0.039$ \\
$K \pi \pi \pi$ & $0.935 \pm 0.043$ & $1.298 \pm 0.076$ & $-0.162 \pm 0.036$ \\
$K \pi \pi^{0}$ & $1.065 \pm 0.036$ & $1.259 \pm 0.060$ & $-0.084 \pm 0.029$ \\
Average & $1.015 \pm 0.024 \pm 0.042$ & $1.280 \pm 0.041 \pm 0.062$ & $-0.116 \pm 0.020 \pm 0.028$ \\
\hline \hline
\end{tabular}

Oscillations between meson and anti-meson, also called mixing, can occur when the flavor eigenstates differ from the physical mass eigenstates. These effects provide a mechanism whereby interference in the transition amplitudes of mesons and anti-mesons may occur. The oscillations are conventionally characterized by two dimensionless parameters $x=\Delta m / \Gamma$ and $y=\Delta \Gamma / \Gamma$. In the absence of CP violation, one has $y_{C P}=y$.

We partly reconstruct the $D$ or $\bar{D}$ which decays to Kev and fully reconstruct the other $\bar{D}$ or $D$ which decays to $K_{S}^{0} / K_{L}^{0}$. When considering $D \bar{D}$ mixing without CPV, $y_{C P}$ then can be determined by $y_{C P}=\frac{L_{K_{S}^{0}}-L_{K_{L}^{0}}}{L_{K_{S}^{0}}+L_{K_{L}^{0}}}$, where $L_{K_{S}^{0} / K_{L}^{0}}=\frac{N_{K_{S}^{0} / K_{L}^{0} \pi^{0}, K e v} / \varepsilon_{K_{S}^{0} / K_{L}^{0} \pi^{0}, K e v}}{N_{K_{S}^{0} / K_{L}^{0} \pi^{0} / \varepsilon_{K_{S}^{0}} / K_{L}^{0} \pi^{0}}}, N_{K_{S}^{0} / K_{L}^{0} \pi^{0}, K e v}\left(N_{K_{S}^{0} / K_{L}^{0} \pi^{0}}\right)$ are the DT(ST) yields, $\varepsilon_{K_{S}^{0} / K_{L}^{0} \pi^{0}, K e v}\left(\varepsilon_{K_{S}^{0} / K_{L}^{0} \pi^{0}}\right)$ are the DT(ST) efficiencies. We obtain $y_{C P}=\left(1.6 \pm 2.5_{\text {stat. }}\right) \%$, which is consistent with previous BESIII measurement with more tag modes [可].

\subsection{Absolute BF measurement of $D^{+} \rightarrow K_{S}^{0} / K_{L}^{0} K^{+}\left(\pi^{0}\right)$ (preliminary)}

Experimental studies of the hadronic decays of charm mesons can shed light on the interplay between the strong and weak forces. In the standard model (SM), the singly Cabibbo-suppressed (SCS) D meson hadronic decays are predicted to exhibit CP asymmetries of the order of $10^{-3}$ [ $[\mathrm{C}]$. Direct CP violation in SCS decays could arise from the interference between tree-level and penguin decay processes. Consequently, any observation of $\mathrm{CP}$ asymmetry greater than $\mathscr{O}\left(10^{-3}\right)$ in any SCS D hardonic decay would be evidence for new physics beyond the SM [ [ $]$ ]. This talk reports the measurements of the absolute BFs and the CP asymmetries of the SCS decays of $D^{+} \rightarrow K_{S}^{0} K^{+}, K_{S}^{0} K^{+} \pi^{0}, K_{L}^{0} K^{+}$and $K_{L}^{0} K^{+} \pi^{0}$. Note that the decay rates of $D^{+} \rightarrow K_{S}^{0} K^{+}\left(\pi^{0}\right)$ and $D^{+} \rightarrow K_{L}^{0} K^{+}\left(\pi^{0}\right)$ are the same because there is no interference of $K^{0}-\bar{K}^{0}$ The measurement of the BF of the two body decay $D^{+} \rightarrow K_{S}^{0} K^{+}$is also helpful for better understanding SU(3)-violating effects in D meson decays [Q].

According to the DT method, the absolute BF for the signal decay is described by $\mathscr{B}_{s i g}=$ $\frac{N_{D T} / \varepsilon_{D T}}{N_{S T} / \varepsilon_{S T}}$, where $N_{D T}\left(N_{S T}\right)$ and $\varepsilon_{D T}\left(\varepsilon_{S T}\right)$ are the yields and efficiencies of DT(ST) modes. With the measured absolute BFs of $D^{+}$and $D^{-}$decays $\left(\mathscr{B}_{\text {sig }}^{+}\right.$and $\left.\mathscr{B}_{\text {sig }}^{-}\right)$, the $\mathrm{CP}$ asymmetry for the decay of interest can be determined by $\mathrm{A}_{C P}=\frac{\mathscr{B}_{\text {sig }}^{+}-\mathscr{B}_{\text {sig }}^{-}}{\mathscr{B}_{\text {sig }}^{+}+\mathscr{B}_{\text {sig }}^{-}}$. The results are shown in Table. ఐ. The $\mathrm{BF}$ of $K_{S}^{0} K$ 
Table 2: Summary of the measured BFs and CP asymmetries, where the first and second uncertainties are statistical and systematic, respectively, and a comparison with the world average value [ए]]

\begin{tabular}{lccccc}
\hline \hline signal mode & $\mathscr{B}_{D^{+}}\left(\times 10^{-3}\right)$ & $\mathscr{B}_{D^{-}}\left(\times 10^{-3}\right)$ & $\overline{\mathscr{B}}\left(\times 10^{-3}\right)$ & $\mathscr{B}_{P D G}\left(\times 10^{-3}\right)$ & $\mathrm{A}_{C P}(\%)$ \\
\hline$K_{S}^{0} K^{ \pm}$ & $3.01 \pm 0.12 \pm 0.08$ & $3.10 \pm 0.12 \pm 0.08$ & $3.06 \pm 0.09 \pm 0.08$ & $2.95 \pm 0.15$ & $-1.5 \pm 2.8 \pm 1.6$ \\
$K_{S}^{0} K^{ \pm} \pi^{0}$ & $5.23 \pm 0.28 \pm 0.24$ & $5.09 \pm 0.29 \pm 0.22$ & $5.16 \pm 0.21 \pm 0.23$ & - & $1.4 \pm 4.0 \pm 2.4$ \\
$K_{L}^{0} K^{ \pm}$ & $3.13 \pm 0.14 \pm 0.10$ & $3.32 \pm 0.15 \pm 0.11$ & $3.23 \pm 0.11 \pm 0.11$ & - & $-3.0 \pm 3.2 \pm 1.2$ \\
$K_{L}^{0} K^{ \pm} \pi^{0}$ & $5.17 \pm 0.30 \pm 0.21$ & $5.26 \pm 0.30 \pm 0.21$ & $5.22 \pm 0.22 \pm 0.21$ & - & $-0.9 \pm 4.1 \pm 1.6$ \\
\hline \hline
\end{tabular}

is consistent with CLEO-c's measurement [प]], the BFs of $K_{S}^{0} K \pi^{0}, K_{L}^{0} K$ and $K_{L}^{0} K \pi^{0}$ are measured for the first time. No evidence of CP asymmetries are found for the 4 SCS decays.

\subsection{BF measurement of some $D^{0(+)} \rightarrow P P$ (preliminary)}

The two-body hadronic decays $D \rightarrow P_{1} P_{2}$ ( $P$ denotes one of pseudoscalar mesons) serve as an ideal testbed to improve the understanding of the weak and strong interactions in decays of charmed mesons. Due to the relatively simple topology, the amplitude of each $D \rightarrow P_{1} P_{2}$ decay can be theoretically derived as a sum of different diagrams based on SU(3)-flavor symmetry [12]]. Comprehensive and improved experimental measurements of the BFs for these decays will help to validate the theoretical calculations. Moreover, these measurements will provide important and complementary data to explore SU(3)-flavor symmetry breaking effects in hadronic decays of the $D$ mesons [13], [44, [15, [16].

Table 3: Measured BFs of $D^{+(0)} \rightarrow P_{1} P_{2}\left(\mathscr{B}_{\text {thiswork }}\right)$, the first and second uncertainties are statistical and systematic, respectively. The world average values $\left(\mathscr{B}_{P D G}\right)$ are also shown.

\begin{tabular}{lcc|lcc}
\hline \hline mode & $\mathscr{B}_{\text {thiswork }}\left(\times 10^{-3}\right)$ & $\mathscr{B}_{P D G}\left(\times 10^{-3}\right)$ & mode & $\mathscr{B}_{\text {thiswork }}\left(\times 10^{-3}\right)$ & $\mathscr{B}_{P D G}\left(\times 10^{-3}\right)$ \\
\hline$D^{+} \rightarrow \pi^{+} \pi^{0}$ & $1.259 \pm 0.033 \pm 0.025$ & $1.24 \pm 0.06$ & $D^{0} \rightarrow \pi^{+} \pi^{-}$ & $1.508 \pm 0.018 \pm 0.027$ & $1.421 \pm 0.025$ \\
$D^{+} \rightarrow K^{+} \pi^{0}$ & $0.231 \pm 0.021 \pm 0.006$ & $0.189 \pm 0.025$ & $D^{0} \rightarrow K^{+} K^{-}$ & $4.233 \pm 0.021 \pm 0.076$ & $4.01 \pm 0.07$ \\
$D^{+} \rightarrow \pi^{+} \eta$ & $3.790 \pm 0.070 \pm 0.076$ & $3.66 \pm 0.22$ & $D^{0} \rightarrow K^{\mp} \pi^{ \pm}$ & $38.98 \pm 0.06 \pm 0.62$ & $39.4 \pm 0.4$ \\
$D^{+} \rightarrow K^{+} \eta$ & $0.151 \pm 0.025 \pm 0.014$ & $0.112 \pm 0.018$ & $D^{0} \rightarrow K_{S}^{0} \pi^{0}$ & $12.39 \pm 0.06 \pm 0.30$ & $12.0 \pm 0.4$ \\
$D^{+} \rightarrow \pi^{+} \eta^{\prime}$ & $5.12 \pm 0.14 \pm 0.21$ & $4.84 \pm 0.31$ & $D^{0} \rightarrow K_{S}^{0} \eta$ & $5.13 \pm 0.07 \pm 0.12$ & $4.85 \pm 0.30$ \\
$D^{+} \rightarrow K^{+} \eta^{\prime}$ & $0.164 \pm 0.051 \pm 0.025$ & $0.183 \pm 0.023$ & $D^{0} \rightarrow K_{S}^{0} \eta^{\prime}$ & $9.49 \pm 0.20 \pm 0.37$ & $9.5 \pm 0.5$ \\
$D^{+} \rightarrow K_{S}^{0} \pi^{+}$ & $15.91 \pm 0.06 \pm 0.33$ & $15.3 \pm 0.6$ & & & \\
$D^{+} \rightarrow K_{S}^{0} K^{+}$ & $3.183 \pm 0.029 \pm 0.067$ & $2.95 \pm 0.15$ & & & \\
\hline \hline
\end{tabular}

ST method is used in this analysis and the $\mathrm{BF}$ of the $D^{+(0)} \rightarrow P_{1} P_{2}$ decay is determined by $\mathscr{B}_{D^{+(0)} \rightarrow P_{1} P_{2}}=\frac{N_{\text {net }}}{2 \cdot N_{D^{+} D-\left(D^{0} \bar{D}^{0}\right)} \cdot \mathcal{E}\left(\cdot \mathscr{B}_{i}\right)}$, where $N_{\text {net }}$ is the background-subtracted signal yields observed in data, $N_{D^{+} D-\left(D^{0} \bar{D}^{0}\right)}$ is the total number of $D^{+} D-\left(D^{0} \bar{D}^{0}\right)$ pairs, $\varepsilon$ is the detection efficiency obtained by the MC simulation, and $\mathscr{B}_{i}$ denotes the BFs of the intermediate resonances $\pi^{0}, \eta, K_{S}^{0}$ and $\eta^{\prime}$. Results are shown in Table B]. Our results are consistent with the world average values within uncertainties and the BFs of $D^{+} \rightarrow \pi^{+} \pi^{0}, \pi^{+} \eta, \pi^{+} \eta^{\prime}, K_{S}^{0} \pi^{+}, K_{S}^{0} K^{+}$and $D^{0} \rightarrow K_{S}^{0} \pi^{0}, K_{S}^{0} \eta, K_{S}^{0} \eta^{\prime}$ are determined with improved precision. The measured BFs for $D^{0} \rightarrow K_{S}^{0} \pi^{0}$ and $D^{+} \rightarrow K_{S}^{0} K^{+}$are 
consistent with those measured using DT technique in Sec. 2.0 and 2.2 , but with better precision. These results will be useful for tests of theoretical calculations and to provide a better understanding of SU(3)-flavor symmetry breaking effects in hadronic decays of the $D^{+(0)}$ mesons.

\subsection{BF measurement of $D^{0(+)} \rightarrow 2 K_{S}^{0}+X$}

In this report, we measure the BFs for the hadronic decays $D^{+} \rightarrow K_{S}^{0} K_{S}^{0} \pi^{+}, D^{0} \rightarrow K_{S}^{0} K_{S}^{0}$, $D^{+} \rightarrow K_{S}^{0} K_{S}^{0} K^{+}$and $D^{0} \rightarrow K_{S}^{0} K_{S}^{0} K_{S}^{0}$. These decays have simpler event topologies and suffer less from combinatorial backgrounds than other decay modes containing two $K_{S}^{0}$ in the final state. The comprehensive or improved measurements of three-body decays will benefit the understanding of the interplay between the weak and strong interactions in multi-body decays where theoretical predictions are poorer than two-body decays. The improved measurements of two-body decays can serve to better explore the contributions of W-exchange diagrams and final-state interactions [ㅁ], [18, [1, [20], as well as SU(3)-flavor symmetry breaking effects [21, [22, [14, 223, 24] in $D$ meson decays. In addition, these measurements will also help to improve background estimations in the precision measurements of $D$ and $B$ meson decays.

(a)

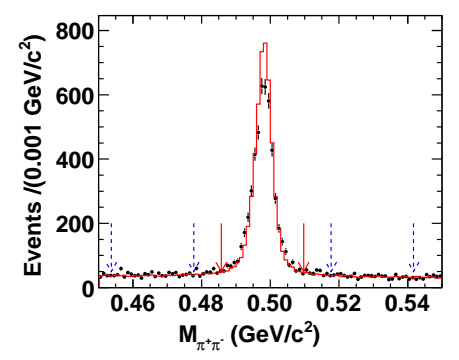

(b)

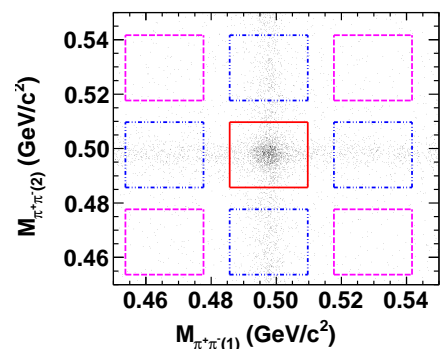

(c)

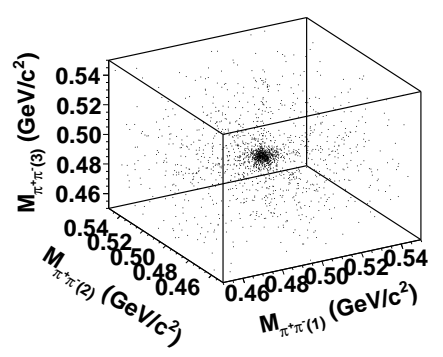

Figure 1: (a) Comparison of the $M_{\pi^{+} \pi^{-}}$distributions of the $D^{0} \rightarrow K_{S}^{0} K_{S}^{0}$ candidate events between data (dots with error bars) and inclusive MC (histogram). The pairs of the solid (dashed) arrows denote the $K_{S}^{0}$ signal (sideband) regions. (b) Distribution of $M_{\pi^{+} \pi^{-}(1)}$ versus $M_{\pi^{+} \pi^{-}(2)}$ for the $D^{0} \rightarrow K_{S}^{0} K_{S}^{0}$ candidate events in data. (c) Distribution of $M_{\pi^{+} \pi^{-}(1)}$ versus $M_{\pi^{+} \pi^{-}(2)}$ versus $M_{\pi^{+} \pi^{-}(3)}$ for the $D^{0} \rightarrow K_{S}^{0} K_{S}^{0} K_{S}^{0}$ candidate events in data.

ST method is used in this analysis. The main peaking background come from similar-finalstates $D$ decay but $\pi^{+} \pi^{-}$pairs do not form $K_{S}^{0}$. Thus, two-dimensional (2D) signal and sideband regions are defined. Figure $\mathbb{W}(\mathrm{b})$ shows the distribution of $M_{\pi^{+} \pi^{-}(1)}$ versus $M_{\pi^{+} \pi^{-}(2)}$ for the $D^{0} \rightarrow$ $K_{S}^{0} K_{S}^{0}$ candidate events in data. The solid box, in which both of the $\pi^{+} \pi^{-}$combinations lie in the $K_{S}^{0}$ signal regions, denotes the $2 \mathrm{D}$ signal region. The dot-dashed (dashed) boxes indicate the $2 \mathrm{D}$ sideband 1 (2) regions, in which one (two) of the $\pi^{+} \pi^{-}$combinations lie in the $K_{S}^{0}$ sideband regions and the others are in the $K_{S}^{0}$ signal region. For the $D^{0} \rightarrow K_{S}^{0} K_{S}^{0} K_{S}^{0}$ decay, $M_{\pi^{+} \pi^{-}(1)}$ versus $M_{\pi^{+} \pi^{-}(2)}$ versus $M_{\pi^{+} \pi^{-}(3)}$ of the candidate events in data is shown in Fig. 1 (c). The region in which all three $\pi^{+} \pi^{-}$combinations lie in the $K_{S}^{0}$ signal regions is taken as the three-dimensional (3D) signal region. The 3D sideband $i(i=1,2,3)$ regions denote those in which $i$ of the three $\pi^{+} \pi^{-}$pairs lie in the $K_{S}^{0}$ sideband regions and the rest are located in the $K_{S}^{0}$ signal regions.

The net numbers of the $D^{0} \rightarrow K_{S}^{0} K_{S}^{0}, D^{+} \rightarrow K_{S}^{0} K_{S}^{0} K^{+}$can be calculated by $N_{\text {net }}=N_{K_{S}^{0} \text { sig }}-$ $\frac{1}{2} N_{\mathrm{sb} 1}+\frac{1}{4} N_{\mathrm{sb} 2}-N_{\text {other }}^{b}$, while $D^{0} \rightarrow K_{S}^{0} K_{S}^{0} K_{S}^{0}$ can be calculated by $N_{\text {net }}=N_{K_{S}^{0} \operatorname{sig}}-\frac{1}{2} N_{\mathrm{sb} 1}+\frac{1}{4} N_{\mathrm{sb} 2}-$ 
$\frac{1}{4} N_{\mathrm{sb} 3}-N_{\text {other }}^{b}$. Then the BF is determined by $\mathscr{B}_{D^{+(0)} \rightarrow f}=\frac{N_{\text {net }}}{2 \cdot \sigma_{D^{+} D^{-}} \mathscr{L} \cdot \varepsilon}$, where $\varepsilon$ is the detection efficiency including the BF of $K_{S}^{0} \rightarrow \pi^{+} \pi^{-}, \mathscr{L}$ is the integrated luminosity of data [25] and $\sigma_{D^{+} D^{-}}\left(D^{0} \bar{D}^{0}\right)$ is the $D^{+} D^{-}\left(D^{0} \bar{D}^{0}\right)$ cross section at the $\psi(3770)$ resonance peak [26]. Finally, we obtain these BFs in Table \&, compare to PDG value [ए]], BF of $D^{+} \rightarrow K_{S}^{0} K_{S}^{0} \pi^{+}$is measured for the first time and others are consistent with previous measurements, but with much improved precision. For more details about this analysis refer to [27].

Table 4: Comparisons of the BFs (in $10^{-4}$ ) measured in this work with the PDG values [س]].

\begin{tabular}{ccc}
\hline \multicolumn{1}{c}{ Decay modes } & \multicolumn{1}{c}{ This work } & PDG \\
\hline$D^{+} \rightarrow K_{S}^{0} K_{S}^{0} K^{+}$ & $25.4 \pm 0.5 \pm 1.2$ & $45 \pm 20$ \\
$D^{+} \rightarrow K_{S}^{0} K_{S}^{0} \pi^{+}$ & $27.0 \pm 0.5 \pm 1.2$ & - \\
$D^{0} \rightarrow K_{S}^{0} K_{S}^{0}$ & $1.67 \pm 0.11 \pm 0.11$ & $1.7 \pm 0.4$ \\
$D^{0} \rightarrow K_{S}^{0} K_{S}^{0} K_{S}^{0}$ & $7.21 \pm 0.33 \pm 0.44$ & $9.1 \pm 1.3$ \\
\hline
\end{tabular}

\section{3. $\Lambda_{c}^{+}$hadronic decays}

\subsection{BR measurement of $\Lambda_{c}^{+} \rightarrow \Sigma^{-} \pi^{+} \pi^{+}$and $\Lambda_{c}^{+} \rightarrow \Sigma^{-} \pi^{+} \pi^{+} \pi^{0}$}

More than 30 years have passed since the $\Lambda_{c}^{+}$baryon was first observed in $e^{+} e^{-}$annihilations by the Mark II experiment [28] and the knowledge of $\Lambda_{c}^{+}$decays remains very poor compared to that for charmed mesons. So far, measured decay modes account for only about $60 \%$ [ए]] of all $\Lambda_{c}^{+}$decays, primarily consisting of modes with a $\Lambda(\Sigma)$ hyperon or a proton in the final state. Decays to the $\Sigma^{-}$hyperon are Cabibbo-allowed and are expected to have large rates. However, no experimental measurements exist except for $\Lambda_{c}^{+} \rightarrow \Sigma^{-} \pi^{+} \pi^{+}$[ए]]. Therefore, searching for additional decay modes with $\Sigma^{-}$in the final state is important to build up knowledge on $\Lambda_{c}^{+}$decays.

DT method is used and the $\Sigma^{-}$hyperon is detected through $\Sigma^{-} \rightarrow n \pi^{-}$. As the neutron is not reconstructed in this analysis, we deduce its kinematic properties by four-momentum conservation. The distributions of $M_{n}$ versus $M_{n \pi^{-}}$for the $\Lambda_{c}^{+} \rightarrow \Sigma^{-} \pi^{+} \pi^{+}$and $\Lambda_{c}^{+} \rightarrow \Sigma^{-} \pi^{+} \pi^{+} \pi^{0}$ candidates in data are shown in Figs. ( (a) and (b), respectively, where clusters corresponding to signal decays are evident. To improve the resolution of the signal mass, as well as to better handle the backgrounds around the $\Sigma^{-}$and neutron mass regions, we determine the signal yields from the distribution of the mass difference $M_{n \pi^{-}}-M_{n}$, since $M_{n \pi^{-}}$and $M_{n}$ are highly correlated. Based on a study of the inclusive MC samples, no peaking backgrounds are expected for these two channels.

The absolute BFs for $\Lambda_{c}^{+} \rightarrow \Sigma^{-} \pi^{+} \pi^{+}$and $\Lambda_{c}^{+} \rightarrow \Sigma^{-} \pi^{+} \pi^{+} \pi^{0}$ are determined by $\mathscr{B}_{\Lambda_{c}^{+} \rightarrow \Sigma^{-} \pi^{+} \pi^{+}\left(\pi^{0}\right)}=$

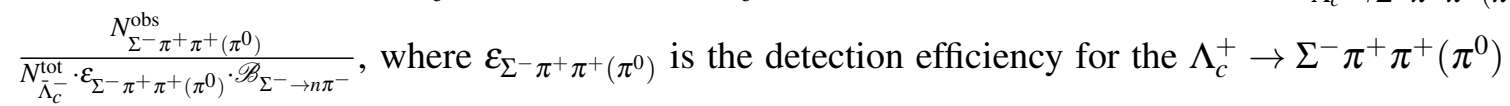
decay with $\Sigma^{-} \rightarrow n \pi^{-}$. The intermediate decay BF of $\Sigma^{-} \rightarrow n \pi^{-}$is included in the denominator. We obtain $\mathscr{B}_{\Lambda_{c}^{+} \rightarrow \Sigma^{-} \pi^{+} \pi^{+}}=(1.81 \pm 0.17 \pm 0.09) \%$ and $\mathscr{B}_{\Lambda_{c}^{+} \rightarrow \Sigma^{-} \pi^{+} \pi^{+} \pi^{0}}=(2.11 \pm 0.33 \pm 0.14) \%$, where the first uncertainties are statistical, and the second are systematic, respectively. This is the first observation of the decay $\Lambda_{c}^{+} \rightarrow \Sigma^{-} \pi^{+} \pi^{+} \pi^{0}$ and the first absolute BF measurement for $\Lambda_{c}^{+} \rightarrow \Sigma^{-} \pi^{+} \pi^{+}$. Our result for $\mathscr{B}_{\Lambda_{c}^{+} \rightarrow \Sigma^{-} \pi^{+} \pi^{+}}$is consistent with and more precise than the previous result [एत]]. For more details about this analysis refer to [ए2]]. 

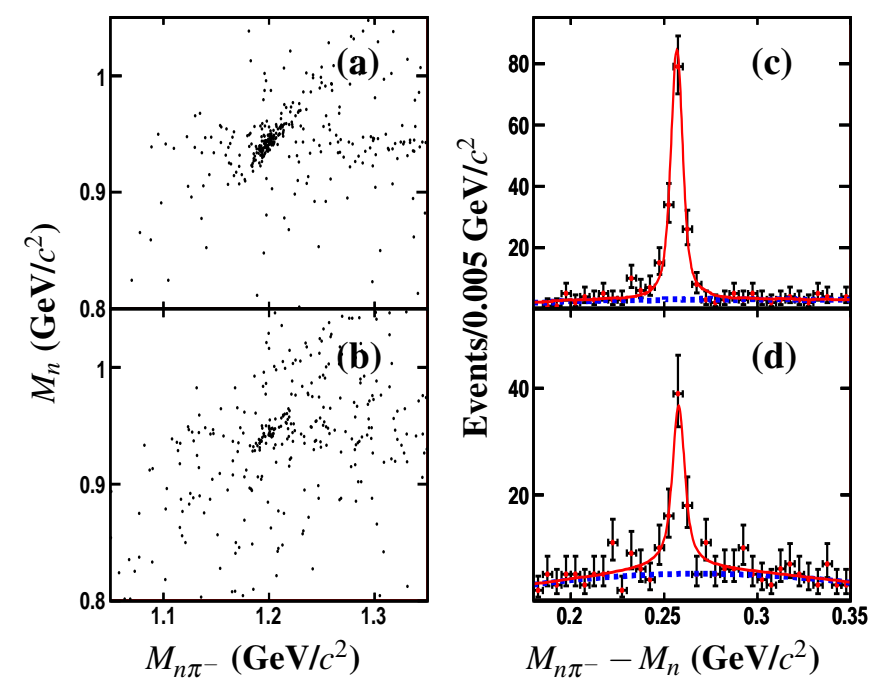

Figure 2: Scatter plots of $M_{n}$ versus $M_{n \pi^{-}}$for candidates in data for (a) $\Lambda_{c}^{+} \rightarrow \Sigma^{-} \pi^{+} \pi^{+}$and (b) $\Lambda_{c}^{+} \rightarrow \Sigma^{-} \pi^{+} \pi^{+} \pi^{0}$. Also shown are fits to the distributions of $M_{n \pi^{-}}-M_{n}$ for (c) $\Lambda_{c}^{+} \rightarrow \Sigma^{-} \pi^{+} \pi^{+}$ and (d) $\Lambda_{c}^{+} \rightarrow \Sigma^{-} \pi^{+} \pi^{+} \pi^{0}$ in data. Solid lines are the results of a complete fit while dashed lines reflect the background components.

3.2 $\Lambda_{c}^{+} \rightarrow p \pi^{+} \pi^{-}$and $\Lambda_{c}^{+} \rightarrow p K^{+} K^{-}$

In contrast to the charmed meson decays, which are usually dominated by factorizable amplitudes, decays of charmed baryons receive sizable nonfactorizable contributions from $W$-exchange diagrams, which are subject to color and helicity suppression. The study of nonfactorizable contributions is critical to understand the dynamics of charmed baryon decays. The SCS decay $\Lambda_{c}^{+} \rightarrow p \pi^{+} \pi^{-}$proceeds via the external $W$-emission, internal $W$-emission and $W$-exchange processes, while the SCS decay $\Lambda_{c}^{+} \rightarrow p K^{+} K^{-}$proceeds via the internal $W$-emission and $W$-exchange diagrams only. Precisely measuring and comparing their BFs may help to reveal the $\Lambda_{c}$ internal dynamics [B]]. A measurement of the SCS mode $\Lambda_{c}^{+} \rightarrow p \phi$ is of particular interest because it receives contributions only from the internal $W$-emission diagrams, which can reliably be obtained by a factorization approach [B]]. An improved measurement of the $\Lambda_{c}^{+} \rightarrow p \phi \mathrm{BF}$ is thus essential to validate theoretical models and test the application of large- $N_{c}$ factorization in the charmed baryon sector [B]], where, $N_{c}$ is the number of colors.

Table 5: Summary of relative and absolute BFs, and comparing with the results from PDG [प]]. Uncertainties are statistical, experimental systematic, and reference mode uncertainty, respectively.

\begin{tabular}{lcc}
\hline \hline Decay modes & $\mathscr{B}_{\text {mode }}$ (This work) & $\mathscr{B}_{\text {mode }}$ (PDG average) \\
\hline$\Lambda_{c}^{+} \rightarrow p \pi^{+} \pi^{-}$ & $(3.91 \pm 0.28 \pm 0.15 \pm 0.24) \times 10^{-3}$ & $(3.5 \pm 2.0) \times 10^{-3}$ \\
$\Lambda_{c}^{+} \rightarrow p \phi$ & $(1.06 \pm 0.19 \pm 0.08 \pm 0.06) \times 10^{-3}$ & $(8.2 \pm 2.7) \times 10^{-4}$ \\
$\Lambda_{c}^{+} \rightarrow p K^{+} K^{-}($non- $\phi)$ & $(5.47 \pm 1.30 \pm 0.41 \pm 0.33) \times 10^{-4}$ & $(3.5 \pm 1.7) \times 10^{-4}$ \\
\hline \hline
\end{tabular}

ST method is used and $\Lambda_{c}^{+} \rightarrow p K^{-} \pi^{+}$is reconstructed as the reference mode. Fig B shows the fit results. In the decay $\Lambda_{c}^{+} \rightarrow p \pi^{+} \pi^{-}$, the peaking background come from the decays $\Lambda_{c}^{+} \rightarrow \Lambda \pi^{+}$ 

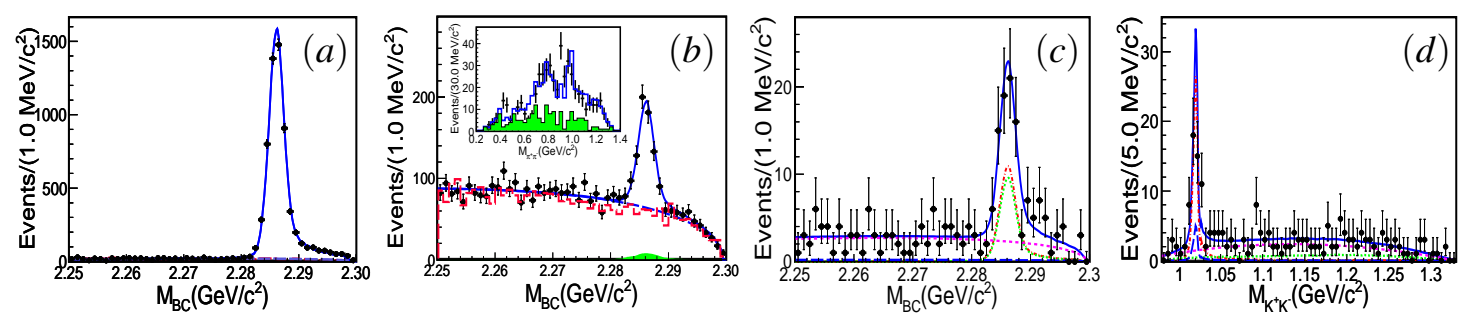

Figure 3: Distributions of $M_{\mathrm{BC}}$ for the decays (a) $\Lambda_{c}^{+} \rightarrow p K^{-} \pi^{+}$, (b) $\Lambda_{c}^{+} \rightarrow p \pi^{+} \pi^{-}$, (c) $\Lambda_{c}^{+} \rightarrow p K^{+} K^{-}$ and (d) $M_{K^{+} K^{-}}$for $\Lambda_{c}^{+} \rightarrow p K^{+} K^{-}$. Points with an error bar are data, the blue solid lines show the total fits, the blue long dashed lines are the combinatorial background shapes, and the red long dashed histograms are data from the $\Delta E$ sideband region for comparison. In (b), the green shaded histogram is the peaking background from the CF decays $\Lambda_{c}^{+}$top $K_{S}^{0}$ and $\Lambda_{c}^{+} \rightarrow \Lambda \pi$. The inset plot in (b) shows the $\pi^{+} \pi^{-}$invariant mass distribution with the additional requirement $|\Delta E|<8 \mathrm{MeV}$ and $2.2836<M_{\mathrm{BC}}<2.2894 \mathrm{GeV} / c^{2}$, where the dots with an error bar are for the data, the blue solid histogram shows the fit curve from PWA, and the green shaded histogram shows background estimated from the $M_{\mathrm{BC}}$ sideband region. In (c) and (d), the blue solid curves are for the total fit results, the red dash-dotted curves show the $\Lambda_{c}^{+} \rightarrow p \phi \rightarrow p K^{+} K^{-}$signal, the green dotted curves show the $\Lambda_{c}^{+} \rightarrow p K^{+} K_{n o n-\phi}^{-}$signal, the blue long-dashed curves are the background with $\phi$ production, and the magenta dashed curves are the non- $\phi$ background.

and $\Lambda_{c}^{+} \rightarrow p K_{S}^{0}$. For the decay $\Lambda_{c}^{+} \rightarrow p K^{+} K^{-}$, we perform a two-dimensional unbinned extended maximum likelihood fit to estimate the $\phi$ and non- $\phi$ process. The results are shown in Table. [1, we present the first observation of the SCS decays $\Lambda_{c}^{+} \rightarrow p \pi^{+} \pi^{-}$and improved (or comparable) measurements of the $\Lambda_{c}^{+} \rightarrow p \phi$ and $\Lambda_{c}^{+} \rightarrow p K^{+} K_{n o n-\phi}^{-}$BFs comparing to PDG values [प]]. The relative BFs with respect to the $\mathrm{CF}$ decay $\Lambda_{c}^{+} \rightarrow p K^{-} \pi^{+}$are measured. The results provide important data to understand the dynamics of $\Lambda_{c}^{+}$decays. They especially help to distinguish predictions from different theoretical models and understand contributions from factorizable effects [B]]. More details can be found in Ref. [B3]].

\section{3 $\Lambda_{c}^{+} \rightarrow p^{+} \eta$ and $\Lambda_{c}^{+} \rightarrow p^{+} \pi^{0}$}

The SCS decays $\Lambda_{c}^{+} \rightarrow p \eta$ and $p \pi^{0}$ have not yet been studied experimentally. These two decays proceed predominantly through internal $W$-emission and $W$-exchange diagrams, which are non-factorizable and not subject to color and helicity suppression in charmed baryon decay. Some theoretical models [34, [35, [36, 137], predict the BFs of these two process under different assumptions (the flavor SU(3) symmetry, FSI) obtaining different results. Therefore, measurements of these BFs will help us to understand the underlying dynamics of charmed baryon decays and distinguish between the different models. Furthermore, the ratio of BFs of these two decays, which is expected to be relatively insensitive to the values of input parameters in the theoretical calculation, is an excellent probe to distinguish between the different models.

We measure the BFs of $\Lambda_{c}^{+} \rightarrow p \eta$ and $p \pi^{0}$ by using ST method, $\eta$ is reconstructed by the two sub-mode $\eta \rightarrow \gamma \gamma$ and $\eta \rightarrow \pi^{+} \pi^{-} \pi^{0}$. The fitting plots can be found in Fig. 团. The corresponding BFs are calculated using $\mathscr{B}_{\Lambda_{c}^{+} \rightarrow p \eta}=\frac{N_{\text {sig }}}{2 \cdot N_{\Lambda_{c}^{+} \bar{\Lambda}_{c}^{-} \cdot \mathcal{E} \cdot \mathscr{B} \text { inter }}}$, where $N_{\text {sig }}$ is the signal yield determined from the $M_{\mathrm{BC}}$ fit, $N_{\Lambda_{c}^{+} \bar{\Lambda}_{c}^{-}}=(105.9 \pm 4.8$ (stat.) \pm 0.5 (syst.) $) \times 10^{3}$ is the total number of $\Lambda_{c}^{+} \bar{\Lambda}_{c}^{-}$pairs in the data [B2], $\varepsilon$ is the detection efficiency estimated by the MC simulation, and $\mathscr{B}_{\text {inter }}$ is the $\eta$ or $\pi^{0}$ decay BF taken from the PDG [ए]]. The factor of 2 in the denominator accounts for 
the charge conjugation of the $\Lambda_{c}^{+}$. The resultant BF of $\Lambda_{c}^{+} \rightarrow p \eta$ is determined to be $\mathscr{B}_{\Lambda_{c}^{+} \rightarrow p \eta}=$ $(1.24 \pm 0.28$ (stat.) \pm 0.10 (syst.) $) \times 10^{-3}$, uncertainties are statistical and systematic, respectively.
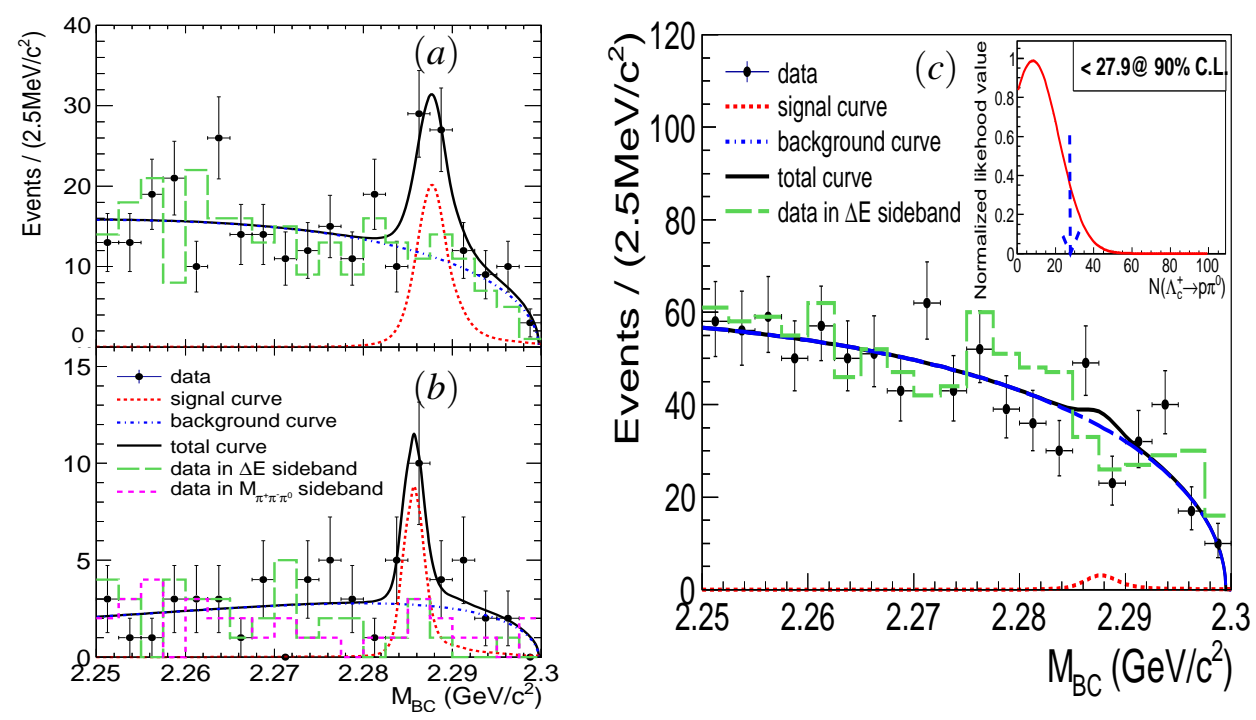

Figure 4: (color online) Simultaneous fit to the $M_{\mathrm{BC}}$ distributions of $\Lambda_{c}^{+} \rightarrow p \eta$ reconstructed with the decay modes (a) $\eta \rightarrow \gamma \gamma$, (b) $\eta \rightarrow \pi^{+} \pi^{-} \pi^{0}$ and (c) $\Lambda_{c}^{+} \rightarrow p \pi^{0}$. The dots with error bars are data, the (black) solid curves are for the best fits, the (blue) dash-dotted curves are for the backgrounds, and the (red) dashed curves are for the signals. The (green) long-dashed histograms and (pink) dashed histogram (in (b) only) are the data in the $\Delta E$ and $M_{\pi^{+} \pi^{-} \pi^{0}}$ sideband region. In (c), the insert shows the normalized likelihood distribution, which includes the systematic uncertainty, as a function of the expected signal yield. The (blue) dashed arrow indicates the upper limit on the signal yield at 90\% C.L.

Since no significant $\Lambda_{c}^{+} \rightarrow p \pi^{0}$ signal is observed, an upper limit on the BF is estimated. The upper limit at the $90 \%$ C.L. on the BF is calculated by substituting $\eta$ with $\pi^{0}$, which is $2.7 \times 10^{-4}$. The corresponding ratio of BFs between the two decays is also calculated to be $\mathscr{B}_{\Lambda_{c}^{+} \rightarrow p \pi^{0}} / \mathscr{B}_{\Lambda_{c}^{+} \rightarrow p \eta}<0.24$, where the common uncertainties are cancelled. The measured BFs and their ratio are compared to the theoretical predictions from different models. More details can be found in Ref. [38]].

\subsection{Measurement of $\Lambda_{c}^{+} \rightarrow \Lambda+X$ (preliminary)}

The inclusive decay $\Lambda_{c}^{+} \rightarrow \Lambda+X$, where $X$ means any possible final state particles, is mediated by the $c \rightarrow s$ Cabibbo-favored transition that dominates the decays of $\Lambda_{c}^{+}$citelbdcx. The measurement of BF of this decay, $\mathscr{B}_{\Lambda_{c}^{+} \rightarrow \Lambda+X}=(35 \pm 11) \%$ [U] ] has a relatively low precision and has not been updated since 1992. It is crucial to improve the accuracy of the BF of the decay $\Lambda_{c}^{+} \rightarrow \Lambda+X$, which allows us to understand the quark structure and decay dynamics of the least massive charmed baryon. In addition, it can also provide an essential input for exploring the decays of the $b$-flavored hadrons involving a $\Lambda_{c}^{+}$in the final states. We also search for the direct CP violation by measuring the charge asymmetry of this inclusive decay $\mathscr{A}_{C P}=\frac{\mathscr{B}_{\Lambda_{c}^{+} \rightarrow \Lambda_{C} X}-\mathscr{B}_{\bar{\Lambda}_{c}^{-} \rightarrow \bar{\Lambda}_{C} X}}{\mathscr{B}_{\Lambda_{c}^{+} \rightarrow \Lambda_{c} X}+\mathscr{B}_{\bar{\Lambda}_{c}^{-} \rightarrow \bar{\Lambda}_{C} X}}$.

DT method is used in this analysis. Only $\Lambda_{c}^{+} \rightarrow p K_{S}^{0}$ and $\Lambda_{c}^{+} \rightarrow p K^{-} \pi^{+}$are tagged to suppress the non- $\Lambda_{c}$ background. Then we search for a $\Lambda$ among the remaining tracks. We extract the signal 
by fitting the $M_{\mathrm{BC}}$ distribution and subtract the sideband. The BF of $\Lambda_{c}^{+} \rightarrow \Lambda_{c} X$ is determined to be $\mathscr{B}_{\Lambda_{c}^{+} \rightarrow \Lambda_{c} X}=\left(38.2_{2.2}^{+2.8} \pm 0.6\right) \%$, where the uncertainties are statistical and systematic, respectively. The precision of the $\mathrm{BF}$ is improved by a factor of 4 , compared to the previous measurement. The $\mathrm{CP}$ asymmetry of the decay $\Lambda_{c}^{+} \rightarrow \Lambda_{c} X$ is obtained by comparing the separate BFs of the charge conjugate decays, which are $\mathscr{B}_{\Lambda_{c}^{+} \rightarrow \Lambda_{c} X}=\left(39.4_{-3.4}^{+4.7}\right) \%$ and $\mathscr{B}_{\bar{\Lambda}_{c}^{-} \rightarrow \bar{\Lambda}_{c} X}= \pm\left(37.8_{-2.9}^{+3.8}\right) \%$. The CP asymmetry is determined to be $\mathscr{A}_{C P}=\left(2.1_{-6.6}^{+7.0}\right) \%$, where the uncertainty is statistical only. The direct $\mathrm{CP}$ violation in this decay is measured for the first time. The precision is limited by statistical uncertainty and no evidence for $\mathrm{CP}$ violation is found.

\section{References}

[1] M. Ablikim et al. (BESIII Collaboration), Nucl. Instrum. Methods Phys. Res. A 614, 345 (2010).

[2] R. M. Baltrusaitis et al. (MARK III Collaboration), Phys. Rev. Lett. 56, 2140 (1986).

[3] I.I. Bigi and H. Yamamoto, Phys. Lett. B 349, 363 (1995).

[4] Z. Z. Xing, Phys. Rev. D 55, 196 (1997).

[5] Q. He et al. (CLEO Collaboration), Phys. Rev. Lett. 100, 091801 (2008).

[6] M. Ablikim et al. (BESIII Collaboration), Phys. Lett. B 744, 339 (2015).

[7] F. Buccella et al. (CLEO Collaboration), Phys. Lett. B. 302, 319 (1993); F. Buccella et al. Phys. Rev. D 51, 3478 (1995); M. Golden and B. Grinstein, Phys. Lett. B 222, 501 (1989).

[8] S. Bianco et al. Riv. Nuovo. Cim. 26N7-8, 1 (2003); A. Le Yaouanc et al. Phys. Lett. B. 292, 353 (1992)

[9] K. Arms et al. (CLEO Collaboration), Phys. Rev. D. 69, 071102 (2004).

[10] K. A. Olive et al. (Particle Data Group) Chin. Phys. C 38, 090001 (2014).

[11] G. Bonvicini et al. (CLEO Collaboration), Phys. Rev. D. 77, 091106(R) (2008).

[12] L.-L. Chau and H.-Y. Cheng, Phys. Rev. D 36, 137 (1987); H.-Y. Cheng and C.-W. Chiang, ibid. 81, 074021 (2010).

[13] K. Waikwok and S. Rosen, Phys. Lett. B 298, 413 (1993).

[14] Y. Grossman and D. J. Robinson, J. High Energy Phys. 2013, 67 (2013).

[15] F.-S. Yu, X.-X. Wang, and C.-D. Lağu, Phys. Rev. D 84, 074019 (2011).

[16] H.-n. Li, C.-D. Lu, and F.-S. Yu, Phys. Rev. D 86,036012 (2012).

[17] X. Y. Pham et al., Phys. Lett. B 193, 331 (1987).

[18] R. E. Karlsen and M. D. Scadron et al., Phys. Rev. D 45, 4113 (1992).

[19] Y. S. Dai et al., Phys. Rev. D 60, 014014 (1999).

[20] J. O. Eeg et al., Phys. Rev. D 64, 034010 (2001).

[21] W. Kwong and S. P. Rosen, Phys. Lett. B 298, 413 (1993).

[22] H. N. Li et al., Phys. Rev. D 86, 036012 (2012).

[23] S. Müller et al., Phys. Rev. D 92, 014004 (2015). 
[24] A. Biswas et al., Phys. Rev. D 92, 014032 (2015).

[25] M. Ablikim et al. (BESIII Collaboration), Chin. Phys. C 37, 123001 (2013); Phys. Lett. B 753, 629 (2016).

[26] G. Bonvicini et al. (CLEO Collaboration), Phys. Rev. D 89, 072002 (2014).

[27] M. Ablikim et al. (BESIII Collaboration), Phys. Lett. B 765, 231 (2017).

[28] G. S. Abrams et al. (Mark II Collaboration), Phys. Rev. Lett. 4410 (1980).

[29] M. Ablikim et al. (BESIII Collaboration), Phys. Lett. B 772, 288 (2017).

[30] H. Y. Cheng, Front. Phys. 10, 101406 (2015).

[31] H. Y. Cheng and C. W. Chiang, Phys. Rev. D 81, 074021 (2010).

[32] M. Ablikim et al. (BESIII Collaboration), Phys. Rev. Lett. 116, 052001 (2016).

[33] M. Ablikim et al. (BESIII Collaboration), Phys. Rev. Lett. 117, 232002 (2016)

[34] K. K. Sharma, R. C. Verma, Phys. Rev. D 55, 7067 (1997).

[35] T. Uppal, R. C. Verna, M. P. Khanna, Phys. Rev. D 49, 3417 (1994).

[36] S. L. Chen, X. H. Guo, X. Q. Li and G. L. Wang, Commun. Theor. Phys. 40, 563 (2003)

[37] Cai-Dian Lü, Wei Wang, and Fu-Sheng Yu, Phys. Rev. D 93, 056008 (2016).

[38] M. Ablikim et al. (BESIII Collaboration), Phys. Rev D 95, 111102 (2017).

[39] J. G. Körner, G. Kramer and J. Willrodt, Phys. Lett. B 279 78, 492 (1978) Erratum: [Phys. Lett. B 81, 419 (1979)] 